\title{
An Optimal Reservation-Pool Approach for Guaranteeing the Call-Level QoS in Next-Generation Wireless Networks
}

\author{
Fei Hu and Neeraj K. Sharma \\ Department of Electrical \& Computer Engineering, Clarkson University \\ P. O. Box 5722, Potsdam, New York 13699-5722, USA \\ \{Huf, sharman\}@clarkson.edu
}

\begin{abstract}
In order to provide the guaranteed mobile QoS (Quality-of-service) for arriving multi-class calls, we need to minimize the dropping rate of handoff calls while at the same time controlling the blocking rate of new calls. This paper proposed a new multi-class call admission control mechanism that is based on dynamically formed reservation pool for handoff requests. The simulation results show that the individual $Q o S$ criteria of multi-class traffic such as the handoff call dropping probability can be achieved within a targeted objective and the new call blocking probability is constrained to be below a given level. The proposed scheme is applicable to channel allocation of multi-class calls over high-speed multimedia wireless networks.
\end{abstract}

\section{Introduction}

Multimedia mobile communications are expected to be the dominant mode of access technology. Besides traditional voice communication, a new range of services such as multimedia, high-speed data, etc. are being offered for delivery over wireless networks. Mobility will be seamless for implementing the blueprint of person's being in contact anywhere and at any time [1-3]. Mobile Quality-of-Service (M-QoS) is a set of performance parameters associated with wireless link such as channel error rate and with mobile units such as Handoff-call Dropping Probability (HDP) and New-call Blocking Probability (NBP). In order to provide higher capacity on the limited radio spectrum, we should use smaller-sized cells such as pico- cells instead of macro- or micro- cells. For such a small cell size, handoff will occur more frequently and make $H D P$ a crucial consideration in $M-Q o S$. Such handoffs involve allocating sufficient resources in each arriving cell to maintain the $Q o S$ needs of the established connections. It is a common practice to give a higher priority to the handoff calls as compared to new calls. On the other hand, giving too much priority to handoff calls will result in an excessive $N B P$. Denying of too many new calls can bring an unacceptable ratio of carried-to-admitted traffic and a unsatisfactory revenue for network providers. Various channel allocation schemes have been proposed to implement handoff prioritization and at the same time not hamper the acceptance of new calls. 
Most of the papers in the literature assume single-class traffic in the cells. The provision of multi-class services (also called multimedia communications) is gaining wide acceptance and will be more ubiquitous in the future wireless and mobile systems.

\subsection{Related Works for Multi-class CAC}

Recently limited work has been reported in the literature regarding CAC schemes in multi-class wireless networks [12-18,20]. In this section we review four different multi-class CAC mechanisms which have been proposed in the literature $[10,16,18,20]$.

S. K. Das et al. [20] developed an integrated framework for $Q o S$ provisioning at a lower layer such as the radio link layer combining a novel $C A C$ strategy. In this paper we will refer to their scheme as Low Layer Control Scheme (LLCS). LLCS can adapt to time-varying and high Bit Error Rate (BER) feature of wireless physical link. LLCS performs $C A C$ on the basis of channel reservation. $Q D$ in [17] is extensively used in situations where call demands exceed the network's capacity. LLCS covers the entire Network-QoS which involves multiple layers. Therefore it does not focus on implementation details of channel reservation and handoff prioritization. In our scheme, we adopt the concept of reservation pool for handoff request reservation. This idea is based on increasingly accurate position predicting technology instead of simple $M H$ classification and destination determination among three neighboring cells in [20]. This improvement means that we can further reduce the over-reserving of wireless bandwidth. Another $C A C$ scheme based on adaptive bandwidth reservation has been proposed by Oliveira et al. in 1998 [16]. We refer it to as Oliver98 scheme. One of the drawbacks of Oliver 98 strategy is that handoff prioritization, a crucial component of $C A C$ mechanism, is based on the concept of Quality Degradation ( $Q D)$. $Q D$ should be equally used for all kinds of calls instead of only handoff calls. Another drawback of Oliver98 strategy is that all of their simulations assume the inter-arrival times of handoff / new calls to follow a geometric distribution, which cannot reflect the actual traffic conditions [18]. The best assumption is general distribution.

Another scheme which we refer to as Potential Resource Estimation Scheme (PRES) is proposed by Ramanathan in [18]. The obvious drawback of PRES is that it shows extremity for handoff prioritization. Handoff prioritization means that we should give handoff calls much higher priority over new calls 1 . However, it does not imply that we should accept all of the handoff calls and consider only the admission control of each arriving new call. If PRES is used in practical systems, it may bring unacceptably high $N B P$ while minimizing $H D P$. This may lead to network providers' unhappiness due to low revenue resulting from low carried traffic.

One Step Prediction Scheme (OSPS) was suggested by Epstein in [10,12-14]. This approach predicts the amount of bandwidth needed in the current cell and each of the

1 In typical cases, the value of $H D P$ is within the range of $10^{-5} \sim 10^{-2}$, and the value of NBP is within the range of $10^{-3} \sim 10^{-1}$. In other words, $H D P$ is generally 100 times larger than NBP in the system. 
neighboring cells for a specified time interval ahead (called One Step) when a new call of any class arrives. One of the drawbacks of OSPS is that it assumes the MH will handoff to all neighboring cells with equal probability when estimating One Step bandwidth. It overestimates the required bandwidth in those neighboring cells and unnecessarily denies many new calls, which makes the NBP unacceptably high when OSPS is applied to practical WATM networks.

\subsection{Contributions to Multi-class $C A C$ Mechanism}

The first contribution to multi-class $C A C$ mechanism is that we give a detailed and practical framework for handoff requests reservation. Our discussion assumes an $a c$ curate next-cell prediction scheme. With the successful application of Kalman filter to Global Position System (GPS) and other position locating systems, a precise next-cell prediction technology will become a reality in the next generation mobile networks. It is unnecessary to assume the $M H$ will handoff to neighboring cells with undeterminable probability such as in Oliver98 strategy. It is also incorrect to regard the probabilities to all neighboring cells as the same value such as in OSPS. The timing relationship is analyzed between handoff request reservation and later handoff call admission. This is very meaningful for practical system implementation. The state transition map is given for our reservation pool mechanism.

Secondly, for guaranteeing the $M-Q o S$ of each class of handoff calls, we propose a new notion of Reservation Ordering $(R O)$ of handoff requests. $R O$ is about the assigning of admission priorities for multi-class calls. However, our admission priority determination is made according to the $M H$ 's time-varying movement behaviors and the desired $M-Q o S$ requirements of the multi-class calls themselves. On the other hand, OSPS determines call priorities based on only calls' $M-Q o S$ profiles. For the computation of $R O$ value, a weighted algorithm is proposed.

Unlike LLCS and Oliver98 strategy, we assume many traffic classes instead of just two classes (real-time and non-real-time). The desired amount of bandwidth and delay requirements for these $Q o S$ profiles can vary greatly. Although PRES and OSPS also assume multi-class traffic, we analyze urgency details of different ATM AAL services instead of simply assuming $K$ classes of mobile users. Such urgency details are used for computing $R O$ value.

Channel shuffling is our modification of bandwidth compression which is proposed in [20]. Because our channel assigning mechanism involves accurate $M H$ identification between handoff request reservation and handoff call admission, we should carry out the shuffling of reservation channels and unoccupied channels at the same time.

Our $C A C$ approach is implemented in a distributed way. The algorithm needs only the signaling information between local $B S$ and $M H$. This method can bring reduced computation load compared to $M S C$-centered control policy.

Table 1 shows the comparisons between the features of our proposed scheme and those of other four schemes.

The rest of this paper is organized as follows. Section 2 describes the detailed procedure for forming handoff request reservation pool which is based on accurate 
next-cell prediction. This is followed by the presentation of $R O$ policy in Section 3 . Section 4 provides our simulation results and corresponding analysis. Finally, we conclude the paper with a discussion of further work in Section 5.

\section{Multi-class Bandwidth Resource Reservation}

\subsection{Next Cell Prediction}

Most of the existing mechanisms for bandwidth reservation and allocation of handoff / new calls assume that we can get the mobility pattern of the $M H$ using profile-based schemes. This assumption may not be valid for practical systems. For example, in wireless ATM network environments, wireless components can be connected to Wide Area Network (WAN), Local Area Network (LAN) or even Home depending on what kind of ATM network is to be accessed. For such varied wired networks, it may not be possible to predict the arrival of $M H$ to some cell since the mobility patterns may not be available. Another drawback for profile-based schemes is that varying traffic conditions suggest that such history-based schemes can never be fully reliable. Therefore we should use real-time position measurements to predict the future path of a moving $M H$. The greatest advantage of future position prediction is that we can determine the next cell which the $M H$ will cross with high accuracy. Therefore we need to reserve wireless resources only in next cell among all of the neighboring cells and eliminate the reservation of excessive bandwidth in those neighboring cells where the sum of arriving probabilities is less than some small value. Taking into consideration the limited radio resources compared to wired part of wireless network, such an advantage is valuable. GPS can estimate the location of a $M H$ with a $95 \%$ probability level within a $100 m$ margin. However, if differential GPS is employed, we can even achieve $3-5 m$ margin [6].

Table 1. Comparisons of different schemes

\begin{tabular}{|c|c|c|c|c|c|}
\hline & LLCSIOW & Obverys (16) & PRESIIS] & OETSIIVI & Our Inoposed \\
\hline $\begin{array}{l}\text { No. gf ckesses } \\
\text { considered }\end{array}$ & ; & $?$ & $\mathrm{UT}$ & & M \\
\hline $\begin{array}{c}\text { Hamilu/f } \\
\text { prioritization } \\
\text { implementation }\end{array}$ & $\begin{array}{c}\text { R - srtur } \\
\text { chands fer } \\
\text { rardoff cals }\end{array}$ & 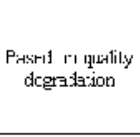 & $\begin{array}{l}\text { Curlutitr ltor } \\
\text { ecmission of } \\
0: 2 y \text { nex calls }\end{array}$ & 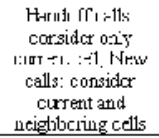 & $\begin{array}{l}\text { Acsuratc and } \\
\text { 1.choulidilly } \\
\text { fommirs } \\
\text { reservation } \mathrm{Fccl}\end{array}$ \\
\hline $\begin{array}{c}\text { Tiaffir. } \\
\text { priorities } \\
\text { consibration }\end{array}$ & No & $\mathrm{No}_{0}$ & No & 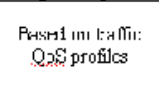 & $\begin{array}{c}\text { baced on rattiz } \\
\text { Qus -ri } 1 \text { mut ifily } \\
\text { kekcwo: }\end{array}$ \\
\hline $\begin{array}{c}\text { Murprimnı. } \\
\text { consiferation }\end{array}$ & No & $\triangle_{0}$ & No & No & $v_{e s}$ \\
\hline $\begin{array}{l}\text { Dynamicalty } \\
\text { Reserve GC }\end{array}$ & Yes & $t^{\prime}=5$ & No & Yes & $\begin{array}{l}\text { Yes ireserration } \\
\text { fintil wilh } 1 \\
\text { rrctcing: }\end{array}$ \\
\hline Use of ftred GC & lNo & $\therefore 0$ & No & liv & Small amour.t. \\
\hline $\begin{array}{c}\text { Destination cell } \\
\text { probability }\end{array}$ & 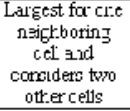 & $\begin{array}{l}0.8 \text { zor one } \\
\text { r.eignoorry zel } \\
\text { an-1 } 0.2 \text { :or the } \\
\text { cum of al other } \\
\text { cells }\end{array}$ & NJ/A & $\begin{array}{c}\text { Equal probabilitics } \\
\text { to al seghbors }\end{array}$ & $\begin{array}{c}\text { Arriuzt.e nest. } \\
\text { scil prcciz:ion } \\
\text { based or. Kalman } \\
\text { filler }\end{array}$ \\
\hline \multirow{2}{*}{ 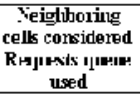 } & $z$ & $\overline{0}$ & $\begin{array}{l}\text { Onty - lie } \\
\text { current cell }\end{array}$ & 6 & 1 (next cel) \\
\hline & No & $\triangle_{0}$ & No & No & $\mathrm{v} \Xi s$ \\
\hline $\begin{array}{c}\text { Irafic } \\
\text { Distrbution }\end{array}$ & Expor:ertal & Goom=tric & Geneal & Exponental & Б̈er:ei \\
\hline
\end{tabular}




\subsection{Determining the Time of Multi-class Handoff Requests Reservation}

In this paper, we give a practical way to determine the Reservation Deadline ( $R D)$ which is a time instance by which bandwidth assignment for the arriving handoff call should be completed. To avoid blind selection of the start point of channel reservation for handoff requests, we define the concept of Core Area $(C A)$ with a radius of size Threshold Distance (TD) in the current cell as shown in Fig. 1 (Right). In $C A$, there is a high probability for the $M H$ to make a dramatic change in its direction and speed. The similar idea is proposed in [6,7]. However, if $M H$ moves beyond $C A$, the chances of sudden change of direction are reduced. Thus we can improve the accuracy of nextcell prediction by using Kalman filter. The reasonable position to start making reservations can be chosen as $O$ shown in Fig. 1 (Right). From the point of view of $R S S$, position $O$ corresponds to the value of RSS1 in the current cell in Fig. 1 (Left). The relationship between the $R S S$ and distance $x$ from the transmitter of the $B S$ is [19]:

$$
R S S_{d B}=-10 \gamma \times \log (x)
$$

where $\gamma$ is the propagation path-loss coefficient.

To determine the value of $R D$, we consider the following two criteria:

(1) The RSS level of current $B S$ drops below a threshold RSS2 so that it is somewhat difficult to keep the communication with $M H$. The position corresponding to RSS2 is shown as $A$ in Fig. 1 (Right).

(2) The RSS level of next-cell $B S$ is stronger than that of the current $B S$ by a given hysteresis margin $\Delta$. That is, we can only serve handoff calls within the shaded $R S S$ range of Fig. 1 (Left).

As can be seen from Fig. 1 (Left), the RSS level meeting condition (1) is on the right of line $A$, while for meeting condition (2) is on the right of line $B$. Thus, to meet both conditions, we have to choose right of line $B$. Therefore, once a $M H$ arrives at position $B$, we should stop the submitting of handoff request immediately. Then the reservation Time Duration $\Omega$ for a $M H$ is from arriving time at position $O$ to arriving time at position $B . \Omega$ can be expressed as:

$$
\Omega=\mathrm{T}_{O B}=t_{B}-t_{O}
$$

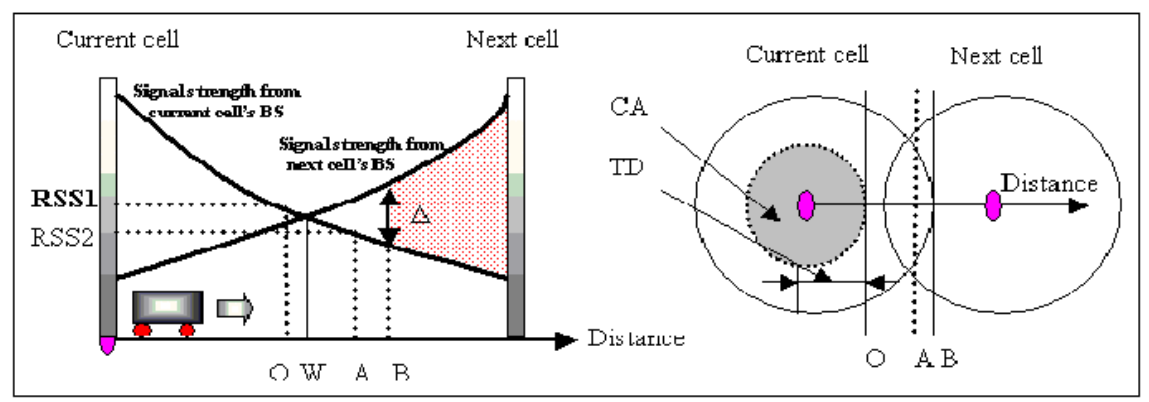

Fig. 1. Time for forming reservation pool (between $\mathrm{O}$ and $A$ ) (Left) RSS point of view (Right) Geometry point of view 
If we consider predominantly walking and stationary users with an average speed of $2 \mathrm{~m} / \mathrm{s}$ and a cell radius of $300 \mathrm{~m}$, which is a common case is wireless ATM campus LAN, the typical value of $\Omega$ is about $5 s \sim 15 s$ [19]. The value of $\Omega$ is important since all of the handoff reservation actions, such as $R O$ and overflow request queuing $R O$ which will be discussed later, should be finished during $\Omega$. Also the values of $Q D T$ and RET (discussed in Section 5) are setup based on the value of $\Omega$.

\subsection{Forming of Multi-class Reservation Pool}

Each handoff $M H$ sends their bandwidth requirements to the $B S$ of next-cell during their own $\Omega$. These handoff request reservations will form a varied-sized pool through marking unoccupied channels from Free to Reserved. As shown in Fig. 2, handoff calls of different classes can reserve highly varying sized Channel Blocks $(C B)$. The term $C B$ comes from the fact that in normal case a handoff call belonging to some class will occupy a series of allocated time slots. The sizes of Free and Occupied bands are also varying since at any time there are always occupied channels released due to calls completion or handoff to another cell. the dark-shaded channel band is marked as GC (Guard Channels).

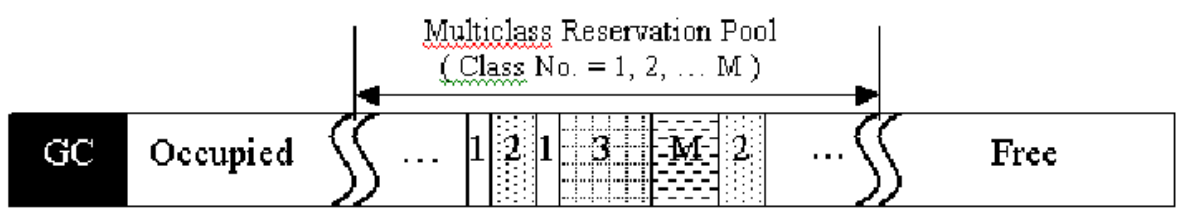

Fig. 2. A snapshot for the channels' status in the current cell

We can draw the State Transition Map (STM) as shown in Fig. 3.

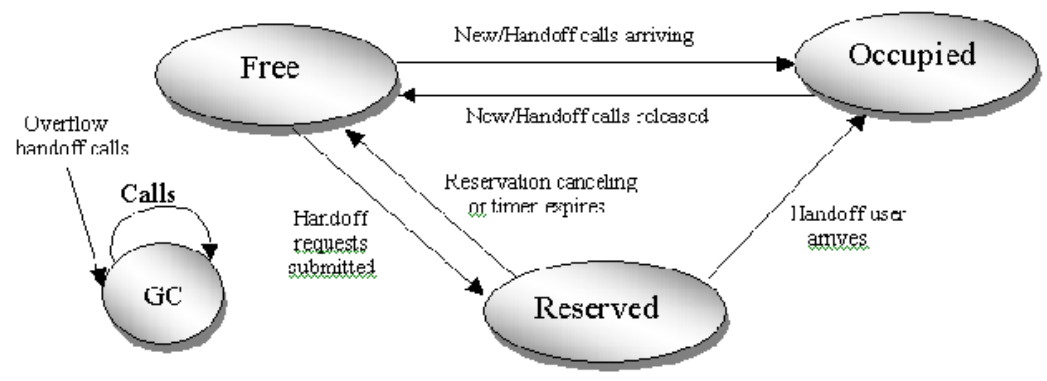

Tig. 3. States Trarsition Mep for the channels with respect to time

\section{Reservation Ordering ( $\mathrm{RO})$ for Multi-class Handoff Calls}

The challenging task of bandwidth assignment for multi-class calls is that we should take into consideration largely different $Q O S$ profiles of each class such as $H D P$, la- 
tency tolerance and desired amount of $W$-EB. For multi-class calls, we should assign each class of calls different priorities during resource allocation, unlike in single class case where all calls are assumed to have the same priority. The role of Reservation Ordering $(R O)$ is to make sure that the service order for each submitted handoff request reservation is maintained.

For determining the $R O$ priority for serving each handoff call, we define a term Class Urgency $(C U)$ which represents the desired serving urgency degree. $C U$ of the coming multimedia calls is determined by their $M-Q o S$ parameters such as delay tolerance and $H D P$. However, $C U$ cannot be used as the only factor for determining the value of $R O$. For example, when a $M H$ is moving almost beyond reservation area (from position $O$ to position $B$ in Fig. 1 (Right)), possibly we should serve this handoff call immediately even though its $C U$ is low since its $R S S$ from the old $B S$ is too weak to continue the communications. In other words, the $R S S$ value can become another factor for determining the $R O$ priority.

Varying speeds of $M H$ can be a serious problem in WATM environment where very rapid fading is common due to its small cell size and low used power. To make the situation worse, the $M H$ in reservation area can wait in traffic jams, traffic lights, or at stop signs. For these cases, it is very improper to assign these $M H$ higher priorities just because their $R S S$ is low. Since $M H$ can travel at different speeds and directions, a faster $M H$ will generally require an earlier handoff than a slower one. Thus $M H$ velocity can become another important factor for determining the $R O$ priority. We can define the $R O$ priority as a two-level weighted scheme:

$$
\begin{gathered}
R O=\left[W_{1} \times(\Delta R S S / \Delta t)+W_{2} \times(R S S)+W_{3} \times(\text { Class Urgency })\right] / 3 \\
\left(W_{1}+W_{2}+W_{3}=1\right)
\end{gathered}
$$

where $\Delta R S S$ / $\Delta t$ reflects the value of $M H$ velocity, and $R S S$ determines the distance of $M H$ from its $B S$ as shown in formula (3). In multi-class network, we can assign $W_{1}, W_{2}$, and $W_{3}$ based on the significance which above-mentioned three factors may have on $R O$. A reasonable weight suite assignment is:

$$
W_{1}=0.1, W_{2}=0.4 \text {, and } W_{3}=0.5
$$

Since $C U$ plays such an important role in multimedia network. Note that we should normalize the value of $\Delta R S S$ / $\Delta t$ and $R S S$ between 0 and 1 . Table 2 shows a possible velocity normalization.

Table 2. A possible velocity normalization result

\begin{tabular}{|c|c|c|c|c|c|}
\hline Average Velocity & $<20 \mathrm{~cm} / \mathrm{s}$ & $1 \mathrm{~m} / \mathrm{s}$ & $10 \mathrm{~m} / \mathrm{s}$ & $20 \mathrm{~m} / \mathrm{s}$ & $>30 \mathrm{~m} / \mathrm{s}$ \\
\hline Practical example & Almost static & Walking & Normal driving & Fast Car & Super Fast \\
\hline Normalized ( $\triangle R S S / \Delta t)$ & 0 & 0.2 & 0.4 & 0.7 & 1 \\
\hline
\end{tabular}

Note that $R O$ depends on two factors. One is $C U$ of handoff calls which is only determined by defined $Q o S$ class. The other is varying mobile behaviors of $M H$. We use velocity $(\Delta R S S / \Delta t)$ and position $(R S S)$ to symbolize the latter factor. This 
scheme is different from $O S P S$ where calls priorities are only determined by class $Q o S$ parameters.

There are already many good ways for measuring $M H$ velocity such as in $[7,8,9,19]$. Thus it is not difficult to obtain the value of $\Delta R S S / \Delta t$.

The following pseudo-code describes the necessary system operations each time a $M H$ handoff request message is sent to the next-cell's $B S$.

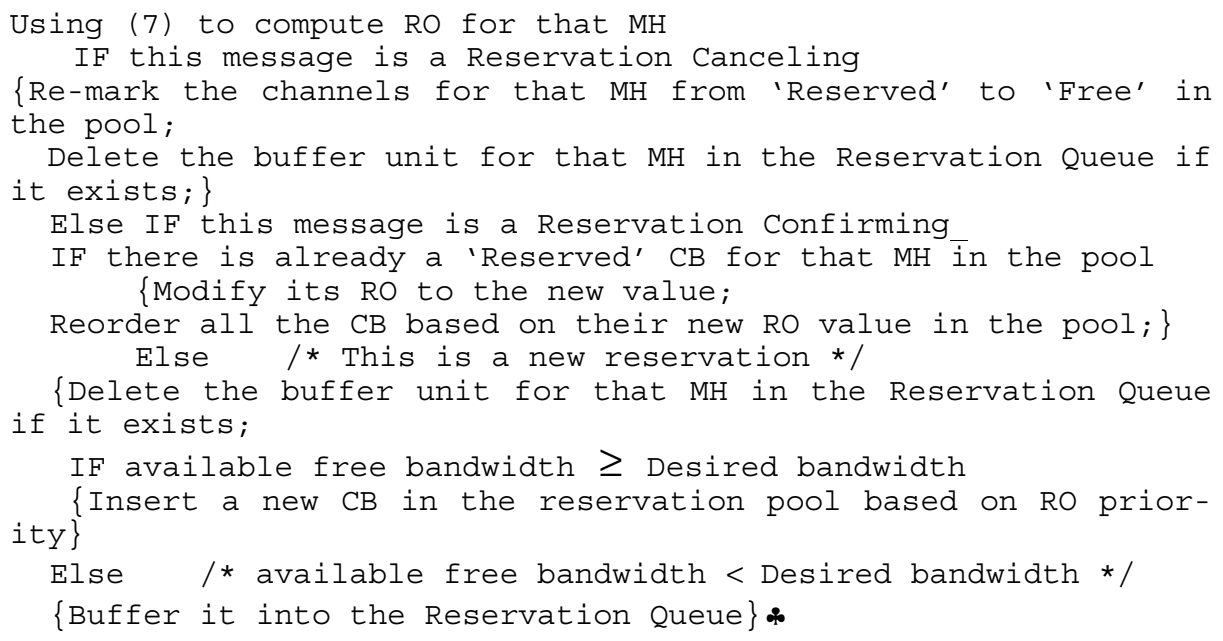

\section{Simulation Experiments}

\subsection{Simulation Model}

Based on the proposed $C A C$ algorithm we built a $\mathrm{C}$-based simulator. In this simulation we choose the total capacity of the current cell as 10,0003 Bandwidth Units $(B U)$. The $B U$ requirements for the five classes of calls are chosen as shown in Table 3.

Table 3. $B U$ requirements for the five classes

\begin{tabular}{|c|c|c|c|c|c|}
\hline Class No. & 1 (Interactive Wideo) & 2 (Videophone) & 3 (Voice) & 4 (WWW) & 5 (E-mail) \\
\hline Desired BU & 30 & 10 & 1 & 10 & 5 \\
\hline
\end{tabular}

The cell radius is assumed to be $500 \mathrm{~m}$, which is a typical size for future WATM system. Three different velocities are assumed: $2 \mathrm{~m} / \mathrm{s}$ (walking), $10 \mathrm{~m} / \mathrm{s}$ (normal-speed car), and $20 \mathrm{~m} / \mathrm{s}$ (high-speed vehicle). Furthermore we assume that the five classes of

3 In this simulation, we choose this capacity value only for testifying the effect of our scheme. As a matter of fact, future WATM or even IMT-2000 should be expected to be able to provide an aggregate transmission capacity of $25 \mathrm{Mb} / \mathrm{s}$ when such systems are offered at frequency bands above $3 \mathrm{GHz}$. 
calls have the same percentages of three velocities in order to emphasize the influence of class urgency on the computation of $R O$. A cluster of seven cells is assumed and each cell keeps contact with its six neighboring cells.

\subsection{The Role of Queue}

Our approach uses a queue for storing overflowing handoff reservations due to the lack of free channels. To investigate the effect of the queue, we assume the same numbers of five class of handoff requests, that is, their percentage within the total handoff requests is $20 \%$ individually. Because handoff congestion happens only when $H T L$ is high, we let $H T L=80 \%$, which makes the $H D P$ almost ten times larger than the $H T L=50 \%$ case.

The HDP results of five classes of handoff calls are shown in Fig. 4. Although each class of handoff calls experience a certain degree of improvement for their HDP due to the introduction of reservation queue, the improvement values are different. It can be seen that class 5 calls have the most dominant decreasing HDP while class 1 calls have the least improvement compared to nо quеие case. A possible explanation for this phenomenon is that class 5 calls have the lowest serving priority among the five classes of calls since only Class Urgency is crucial for computing the value of $R O$ after the elimination of other factors such as mobile movements. Since the percentage of class 5 users is the same as other classes, class 5 calls will have the largest probability for being buffered into reservation queue. Therefore they benefit the most from reservation queue.

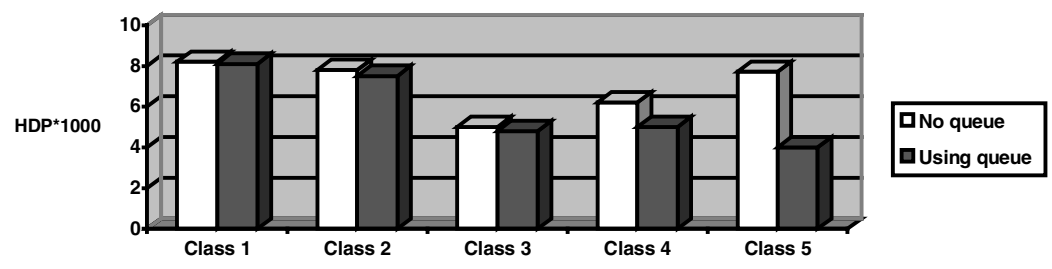

Fig. 4. The importance of reservation queue

\subsection{The Importance of Determining Multimedia Servicing Prioritization}

If we assume that $M H^{\prime}$ 's position and velocity cannot influence much on the $R O$ of each handoff call except for the $C U$ of each class we can see the effect of $R O$ on improving HDP of each class of handoff calls.

We only consider two classes of calls: class 1 and class 5, since class 1 calls have the most crucial urgency requirements while class 5 calls have the least urgency requirements. Two important cases are considered: light handoff load $(H T L=25 \%)$ and

4 This can be done by assuming each class of calls have the same percentage of all types of moving users such as pedestrians and cars. 
heavy handoff load $(H T L=75 \%)$. The reason for choosing these two extreme cases is that we may see the effect of $R O$ on $H D P$ more clearly.

Figure 5 (a) (d) are our simulation results. The $X$-axis represents the percentage of a given class of calls among all handoff calls. It varies from $20 \%$ to $100 \%$. The $Y$ axis is the value of HDP multiplied by 10,000 . It can be seen that HDP of Class 1 calls decreases when $R O$ is adopted. Although in light handoff load case, the reduction is not very obvious (Fig. 5 (a)), in heavy handoff load case the effect of $R O$ is very dominant (Fig. 5 (b)). This is not a surprising result since $R O$ can assign class 1 calls the highest priority when only $C U$ is considered.

Unfortunately, HDP increases for class 5 calls (Fig. 5 (c) and (d)), especially in heavy handoff load case (Fig. 5 (d)). This is because class 5 calls get the lowest priority when their $R O$ is compared to other classes. When the network is under congestion, the class 5 calls have the highest probability for being dropped among the five classes.

For dealing with this problem, we can use the crossover ATM switch to buffer those delay-insensitive class 5 ATM cells. When the handoff connection is rerouted from the old path to a new path, a crossover switch should be found out using fast searching algorithm [5]. Thus, the down-link data stream can be stored in the buffer of this switch.

\section{Conclusions and Future Work}

This paper addressed the problem of providing $M-Q o S$ guarantee for multi-class calls in the WATM network. The network is assumed to be able to accurately predict nextcell which the $M H$ will cross. This assumption is reasonable for the developing mobile position system such as GPS. A multi-weighted algorithm for computing priorities of handoff requests was proposed in order to serve arriving multi-class calls with highly diverse $Q o S$ parameters. A dominant feature of our approach is combining practical handoff behaviors with the call admission procedure. This includes the $R O$ computation and the notion of three timers. Several important considerations for practical system implementation were discussed in this paper.

In the introduction of this paper we mentioned that we focus on the $L C A$ mechanism instead of $C C A$ mechanism. However, there is close relationship between these two mechanisms. A typical example is Channel Borrowing Mechanism (CBM) [4]. $C B M$ states that the whole capacity of any cell is not a fixed value. Each cell only keeps a set of nominal channels (less than FCA case) and can borrow free channels from its neighboring cells to accommodate new calls. Thus, the $N B P$ can be further decreased. One of our future tasks is combining the $C B M$ with our proposed approach to investigate the improvement of $N B P$. Another future task is to derive analytical models to evaluate the performance of our $C A C$ scheme. As shown in Fig. 1, this paper provides a reservation-based call admission strategy for guaranteeing the network $Q o S$. Further work in this area will include translating the high-level resource allocations into scheduling at the low levels such as MAC layer so as to map the network $Q o S$ to MAC-oriented QoS. 


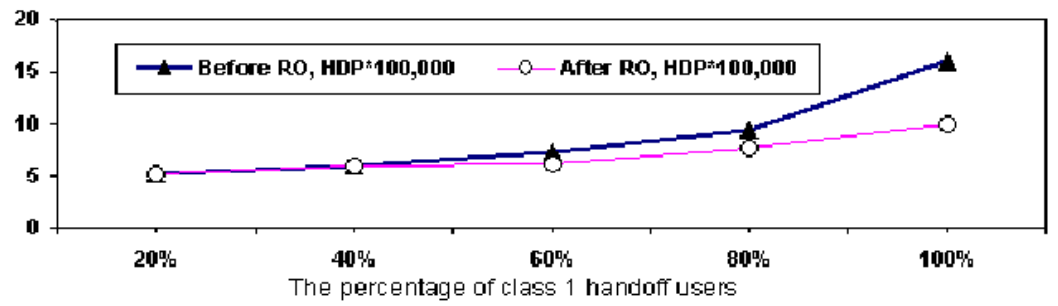

(a) $H D P \times 100,000$ for Class I with $H T L=25 \%$

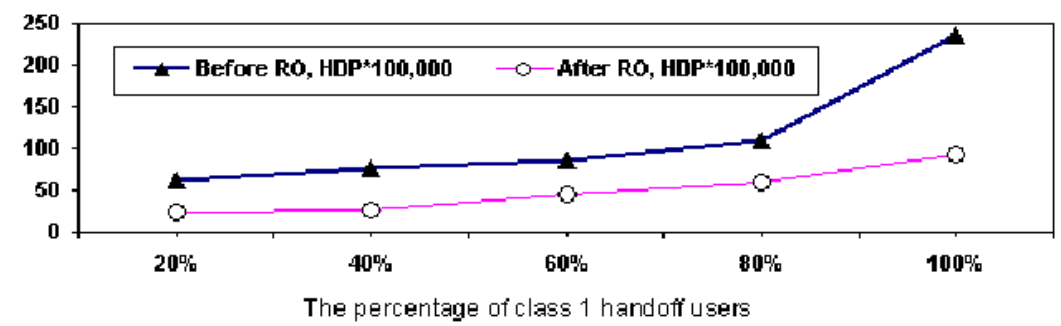

(b) $H D P \times 10,000$ for Cass I with $H T L=75 \%$

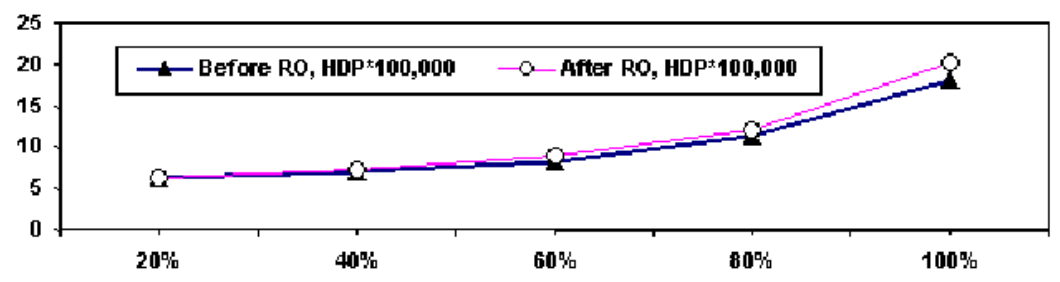

The percentage of class 5 handoff users

(c) $H D P \times I 00,000$ for $Q_{a s s} S$ with $H T L=25 \%$

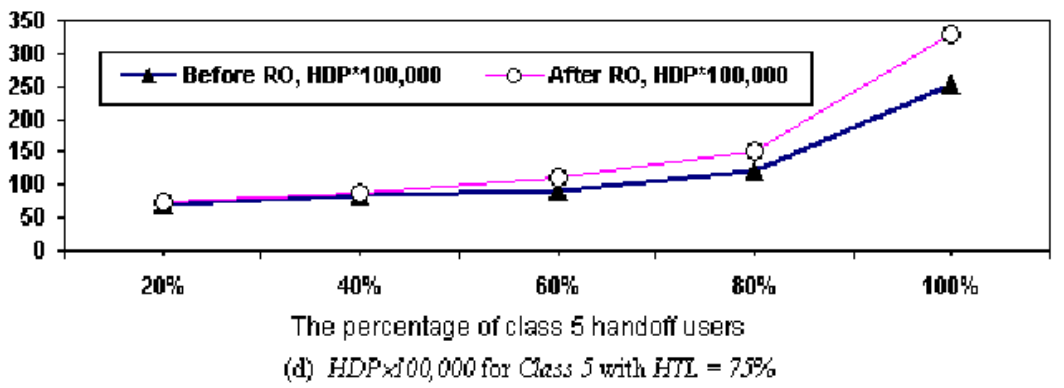

Fig. 5. The influence of $R O$ on $H D P$

\section{References}

1. Mansoor Shafi, et al., "Wireless Communications in the Twenty-First Century: A Perspective," Proceedings of the IEEE, vol. 85, no. 10, pp. 1622-1637, October 1997. 
2. Upkar Varshney and Ron Vetter, "Emerging Mobile and Wireless Networks," Communications of the ACM, vol. 43, no. 6, pp. 73-81, June 2000.

3. Lajos Hanzo, "Bandwidth-Efficient Wireless Multimedia Communications," Proceedings of the IEEE, vol. 86, no. 7, pp. 1342-1380, July 1998.

4. I. Katzela and M. Naghshineh, "Channel Assignment Schemes for Cellular Mobile Telecommunication Systems: A comprehensive Survey," IEEE Personal Communications, pp. 10-31, June 1996.

5. C-K Toh, "Wireless ATM and Ad-Hoc Networks : Protocols and Architectures," pp. 8898, Kluwer Academic Publishers, 1997 (ISBN: 079239822X).

6. Ming-Hsing Chiu and Mostafa A. Bassiouni, "Predictive Schemes for Handoff Prioritization in Cellular Networks Based on Mobile Positioning," IEEE J. Select. Areas Commun., vol. 18, no. 3, March 2000.

7. Tong Liu, et al. "Mobility Modeling, Location Tracking, and Trajectory Prediction in Wireless ATM Networks," IEEE J. Select. Areas Commun., vol. 15, pp. 1208-1225, Sept. 1997.

8. Tong Liu , "An Optimal Self-Learning Estimator for Predicting Inter-Cell User Trajectory in Wireless Radio Networks," IEEE Globecom, 1997.

9. Martin Hellebrandt, et al., "Estimating Position and Velocity of Mobiles in a Cellular Radio Network," IEEE Trans. on Vehicular Technology, vol. 46, no. 1, Feb. 1997.

10. B.Epstain and M.Schwartz, "Reservation strategies for multimedia traffic in a wireless environment," Proc. 1995 IEEE 45 th Vehicular Technology Conf., pp. 165-169, July 1995.

11. Larry L. Peterson and Bruce S. Davie, "Computer Networks: A System Approach," Morgan Kaufmann Publishers, San Francisco, California, pp. 649-662, 2000. (ISBN: 1558605142)

12. Bracha M. Epstein and Mischa Schwarz, "Predictive QoS-Based Admission Control for Multiclass Traffic in Cellular Wireless Networks," IEEE JSAC, vol. 18, no. 3, pp. 523534, March 2000.

13. Bracha M. Epstein and Mischa Schwarz, "QoS-based predictive admission control for multi-media traffic," in Broadband Wireless Communications, M. Luise and S. Pupolin, Eds. Berlin, Germany: Springer-Verlag, pp. 213-224, 1998.

14. Bracha M. Epstein, "Resource Allocation Algorithms for Multi-Class Wireless Networks," Ph.D. dissertation, Columbia Univ., New York, 1999.

15. C. Chao and W. Chen, "Connection Admission Control for Mobile Multi-Class Personal Communications Networks," IEEE JSAC, vol. 15, no. 8, pp. 1618-1626, October 1997.

16. Carlos Oliveira, et al., "An Adaptive Bandwidth Reservation Scheme for High-Speed Multimedia Wireless Networks," IEEE JSAC, vol. 16, no. 6, pp. 858-873, August 1998.

17. K. Seal and S. Singh, "Loss profiles: A quality of service measure in mobile computing," Wireless Networks, vol. 2, no. 1 , January 1996.

18. Parameswaran Ramanathan, et al., "Dynamic Resource Allocation Schemes During Handoff for Mobile Multimedia Wireless Networks," IEEE JSAC, vol. 17, no. 7, pp. 12701283, July 1999.

19. Howard G. Ebersman and Ozan K. Tonguz, "Handoff Ordering Using Signal Prediction Priority Queuing in Personal Communication Systems," IEEE Trans. on Vehicular Technology, vol. 48, no. 1, pp. 20-35, Jan. 1999.

20. S. K. Das, et al., "A Call Admission and Control Scheme for Quality-of-Service (QoS) Provisioning in Next Generation Wireless Networks," Wireless Networks 6, pp. 17-30, 2000. 